\title{
Contribuições de Modelos Baseados em Agentes (MBA) para gestão de sistemas hídricos urbanos
}

\section{Diana Jimena Monsalve Herrera Conceição de Maria Albuquerque Alves}

\section{RESUMO}

Os atuais Planos de Emergência (PE) para enfrentamento de escassez hídrica contam com o uso de sistemas de apoio a decisão que integram modelos matemáticos para representação do comportamento de sistemas hídricos frente a cenários de precipitação e de uso e ocupação do solo. A disponibilidade hídrica resultante dessas simulaçôes tem sido calculada considerando a demanda como variável de entrada (externa) e sem avaliação do nível de cooperação a ser apresentado pelos consumidores de água frente a medidas e ações de gestão presentes nos PE. Este artigo apresenta fundamentos, aplicaçóes e perspectivas do uso de Modelos Baseados em Agentes na gestão da demanda de sistemas hídricos urbanos. É apresentada a concepçáo de um MBA para gestáo da demanda de água na Regiáo Administrativa de Brazlândia, no Distrito Federal brasileiro. No modelo concebido, cada agente (reativo) representa uma unidade familiar e suas decisóes foram delineadas com uso da metodologia PAGE (Perceptions, Actions, Goals, Environmental). Os agentes foram categorizados por renda e o ambiente urbano é percebido pelos agentes em período seco ou chuvoso. A parametrizaçáo do comportamento dos agentes foi realizada por meio de questionários e permitiu obter um modelo conceitual implementado em plataforma multiagente. A aplicação do modelo possibilitou identificar padróes de comportamento de usuários urbanos de água em Brazlândia por categoria de renda frente a diferentes medidas de gestão.

Palavras-chave: Modelos Baseados em Agentes (MBA), Sistemas Complexos Adaptativos (SCA), Gestão da Demanda de Água, Sistemas Hídricos Urbanos.

\section{ABSTRACT}

The usual Emergency Plans for urban water resources shortage consider the use of decision support systems based on mathematical models that represent the hydrologic response of the system for different precipitation regimes and land use changes. The resulting water availability in these models has considered the urban water demand as an external variable (input) not sensitive to the level of cooperation of the water consumers in reducing their levels of consumption. This work presents the bases, applications and perspectives of the use of Agent Based Models (ABM) in urban water resources system management. It presents the conceptualization of a ABM applied to the urban water management of Brazlandia, in the Federal District of Brazil. The model uses reactive agents to represent households and their behavior and decisions were designed using the methodology PAGE (Perceptions, Actions, Goals, Environmental). The agents were classified as low, medium and high income. The environment was represented by raining and dry seasons. The conceptual model was implemented and parameterized based on field investigations and interviews in selected urban areas of Brazlandia. The application of the ABM showed some possibilities of urban water management improvements when considering the cooperation and behavior of water users in simulation models.

Keyword: Agent Based Models (MBA), Complex Adaptive Systems (CAS), Water Urban Resources, Decision Support Systems 


\section{INTRODUÇÁO}

A gestão de sistemas hídricos urbanos se desenvolve com observância a processos hidrológicos, econômicos, políticos e sociais. A complexidade das leis que governam esses processos e as açóes que resultam das interaçóes entre eles proporcionam propriedades aos sistemas hídricos urbanos que permitem sua caracterização como sistemas complexos adaptativos (MITCHELL, 2006; SICHMAN, 2015). A dinâmica e a possibilidade de aprendizado, de evolução e de adaptação de atores envolvidos no funcionamento desses sistemas (consumidores, companhias de saneamento, agências reguladoras) resultam em comportamentos não determinísticos que caracterizam sistemas complexos. Além disso, o comportamento humano introduz uma complexidade a mais nas questóes de gestáo de sistemas hídricos, devido a diferentes preferências, fraquezas, desvios de percepçáo, pluralidade e diversidade que influenciam o uso (demanda) da água.

Considerar esses elementos na simulação do comportamento de sistemas hídricos urbanos pode contribuir para definição de programas (medidas e açôes) de gestão mais efetivos e adaptados à realidade local (GIACOMONI; KANTA; ZECHMAN, 2013; KANTA; ZECHMAN, 2014). As características dos sistemas complexos podem ser identificadas nos sistemas hídricos pois, além de outras propriedades, contam com uma grande quantidade de componentes (agentes, entidades, etc.) e subsistemas que interagem de forma emergente. Nesse contexto é relevante reconhecer que se está lidando com sistemas complexos na gestáo de recursos hídricos, pois estes sofrem mudanças pela intervenção de diferentes agentes ou do ambiente existindo a necessidade de adaptação.

O comportamento complexo dos sistemas hídricos muitas vezes dificulta a gestão do sistema para solucionar problemas como a falta de disponibilidade do recurso (sistema físico e natural) que resultam em conflitos pelo uso da água, problemas de articulação institucional, e as altas demandas de água, portanto se faz importante considerar um tipo de gestáo não convencional que se adapte às mudanças que apresentam este sistema complexo.

A gestão adaptativa da água considera a complexidade dos sistemas a serem gerenciados e os limites de prever e controlar. Isto implica numa abordagem de gestão integrada que adote uma perspectiva sistêmica ao oposto de lidar com problemas individuais e isolados (KEUR et al., 2010).

Os sistemas hídricos abordados como sistemas complexos adaptativos-SCA podem ser simulados mediante modelos baseados em agentes-MBA (HOLLAND, 1995) que têm suas raízes na área da robótica, no campo da inteligência artificial e nos sistemas multiagentes. Porém, os MBA não podem ser concebidos só como simulaçóes com agentes artificiais, pois, suas principais raízes estão em modelar o comportamento social e a tomada de decisóes individuais (de seres vivos em geral, incluindo o ser humano) que representam a interação social, colaboração e comportamento de grupo (BONABEAU, 2002; MACAL; NORTH, 2010).

Os MBA conseguem modelar sociedades artificiais que permitem a concepçáo de entidades de manejo dos recursos naturais (DUCROT et al., 2004), dando importância à dinâmica de níveis de organização (dos consumidores dos recursos e dos provedores) que atuam através de regras e agentes (RAMMEL; STAGL; WILFING, 2007).

Este artigo tem como objetivo apresentar a concepção de um modelo de representação do comportamento de consumidores domésticos de água com base em modelos baseados em agentes e identificar contribuiçóes de sua aplicação para melhoria da gestáo de sistemas urbanos de abastecimento de água. Para tanto, faz-se uma descrição dos principais conceitos relacionados a sistemas complexos adaptativos ao longo de 3 seçóes, sendo a primeira dedicada à descrição dos sistemas complexos adaptativos e suas características principais. $\mathrm{Na}$ segunda seção, são apresentados fundamentos para modelagem baseada em agentes, descrevendo suas características principais. $\mathrm{Na}$ terceira seção são apresentadas aplicaçóes de modelos baseados em agentes no âmbito da gestão adaptativa dos recursos hídricos considerados como sistemas complexos e caracterizando o comportamento dos agentes.

\section{SISTEMAS COMPLEXOS ADAPTATIVOS-SCA}

Os sistemas complexos adaptativos (SCA) são caracterizados em geral por interaçóes mútuas entre os sistemas ecológico, tecnológico e socioeconômico 
em várias escalas. Sistemas complexos são constituídos por uma rede de componentes relativamente simples (se comparados ao comportamento coletivo), sem controle central, nos quais é exibido um comportamento complexo emergente (MITCHELL, 2006).

Não há viabilidade em projetar ou representar sistemas complexos em detalhe. Eles são compostos por agentes que interagem criando propriedades emergentes, qualidades e padróes de comportamento. Quando os agentes representam seres humanos que não trabalham com regras estritas, seu comportamento não tem previsibilidade (MITLETON-KELLY, 2003).

Os SCA estão baseados em comportamentos complexos, resultado das interaçóes entre os componentes do sistema (ou agentes) ou, entre os componentes do sistema (ou agentes) e o ambiente. Através dessa interaçáo e do aprendizado com o ambiente, o sistema modifica seu comportamento para se adaptar às mudanças (RAMMEL; STAGL; WILFING, 2007).

De uma maneira geral, os SCA apresentam as seguintes características: (SICHMAN, 2015):

- Emergência e retroalimentação (feedback): É um padrão de comportamento que ocorre conforme aumentam as interaçóes das entidades e a complexidade do sistema, e que pode ser significativamente diferente do comportamento do sistema em níveis mais baixos de complexidade (BLASKOVICH, 2014).

- Heterogeneidade: esta característica resulta de uma propriedade intrínseca (ou previamente adquirida) do agente, que tem suas próprias preferências.

- Robustez: determina a capacidade do sistema de manter o comportamento depois de remover algum componente do subsistema ou sofrer alguma perturbação. Esta propriedade é desejável em sistemas influenciados por políticas públicas, para que não sejam alterados por açóes isoladas de indivíduos ou por variaçóes no sistema.

- Resiliência: Esta propriedade indica a capacidade adaptativa para se reorganizar numa estrutura diferente mantendo a função do sistema. A resiliência está relacionada com o feedback que leva à aprendizagem e auto-organização dos atores conforme surgem novas condiçóes.
- Não linearidade: a interação dos indivíduos raramente segue regras lineares, além disso, cada ator tem comportamentos e interaçóes diferentes.

- Sistemas abertos: os indivíduos entram e saem dinamicamente do sistema sem uma governança global.

As características listadas acima podem ser identificadas nos sistemas de recursos hídricos especialmente quando os atores envolvidos na gestão desses sistemas são incorporados, ou seja quando o sistema social é inserido na simulação do funcionamento do sistema hídrico. Em geral, um sistema de recursos hídricos é formado por subsistemas que se relacionam e interconectam para formar uma unidade. São exemplos disso o sistema físico de abastecimento de água (Manancial, ETA, Rede de distribuição), o sistema natural (variáveis biofísicas: solo, cobertura vegetal, precipitação etc.) e o sistema socioeconômico formado pela população e suas atividades econômicas, hábitos, cultura, valores e padrôes de uso dos recursos hídricos. No caso de sistemas hídricos, as instituiçôes que compóem o sistema de gestão dos recursos hídricos (companhias de saneamento, agências reguladoras, companhias de gestão, comitês de bacia hidrográfica etc.) também fazem parte do sistema social que interage com o sistema hídrico e que pode interferir no seu funcionamento. $\mathrm{Na}$ modelagem do sistema hídrico, essas instituiçôes são representadas por atores com papéis definidos que podem ser representadas por agentes integrantes do sistema multi agente a ser simulado por MBA.

A integração do subsistema socioeconômico à operação do sistema hídrico implica na representação do comportamento dos agentes sociais e econômicos frente ao funcionamento do sistema hídrico. Tal requisito eleva o nível de complexidade do sistema hídrico, pois o comportamento dos agentes tanto individual quanto coletivamente apresenta desafios à sua predição e representação. A interdependência e relação entre os diferentes subsistemas que formam o sistema de recursos hídricos e as interações entre os agentes ou entidades de cada um deles resultam em comportamentos complexos de difícil representação.

Podem-se identificar características de sistemas hídricos próprias dos sistemas complexos, tais como: 
- Emergência, que pode ser observada na disponibilidade de água para consumo humano que dependerá do resultado da interação dos atores (companhia de saneamento, reguladores, usuários etc.) criando padróes de comportamento afetando o balanço demanda-oferta. Estas propriedades emergentes retroalimentam-se afetando as decisóes individuais referentes ao uso da água;

- Heterogeneidade, que pode ser observado no comportamento diverso dos usuários referente ao uso da água, pois dependendo das suas necessidades e preferências, ter-se-á um consumo de água excessivo ou não, que pode afetar o sistema hídrico;

- Resiliência, representada na capacidade dos usuários da água em se adaptar às políticas de consumo ou problemas de escassez;

- Não linearidade, pois balanço hídrico do sistema e as interaçóes dos atores entre si e entre eles e o sistema não seguem um padrão linear de comportamento.

Os SCA podem ser simulados mediante diferentes modelos, entre eles existe os modelos baseados em agentes- MBA (HOLLAND, 1995) que simulam a interação entre as partes permitindo entender as consequências das decisôes sobre os diferentes atores e seus comportamentos (AKHBARI; GRIGG, 2013). Outro modelo utilizado é o Cellular automata que é composto por quatro elementos principais: uma rede, um conjunto de estados permitidos e vizinhança, definidos pela estrutura e regras de transição considerando um componente temporal (BATTY; XIE; SUN, 1999).

O comportamento adaptativo e evolutivo dos métodos utilizados em sistemas complexos permite que os indivíduos modelados não só mudem seu comportamento uma vez, frente a uma nova política, mas que se adaptem e evoluam seu comportamento ao longo do tempo (RAND, 2015).

\section{MODELOS BASEADOS EM AGENTES-MBA}

Conforme Bonabeau (2002), na modelagem baseada em agentes o sistema é modelado como um conjunto de entidades autônomas chamadas agentes e que podem possuir níveis diferenciados de autonomia e de tomada de decisão. Os MBA que buscam representar comportamentos sociais não são trabalhados para predizer o pensamento dos atores (skateholders ou gestores) no momento de tomar decisôes. Em contrapartida a modelagem pode predizer o comportamento do sistema submetido a certos parâmetros e condiçóes iniciais que incluem as decisóes e comportamentos dos agentes, sendo uma ferramenta útil para comparar estratégias, explorando atributos complexos e características dos sistemas (BONABEAU, 2002; ZECHMAN, 2015).

Um agente é basicamente um elemento para análise de sistemas (RUSSELL; NORVIG, 1995) e é qualquer entidade que percebe seu ambiente através de sensores e age sobre ele (RUSSELL; NORVIG, 2002). O ambiente (sistema) em que o agente irá atuar delimitará a abrangência de suas percepções e açôes.

Segundo Wooldridge e Jennings (1995) os agentes podem apresentar as seguintes características:

- Autonomia: tem a capacidade de operar, seguir instruções e tomar decisóes sem intervenção de outros, tendo o controle de suas açóes. Quando um agente aprende por experiência e altera seu comportamento, tem autonomia funcional. Dessa maneira, os agentes são mais flexíveis adaptando-se a novas condiçóes do ambiente (RUSSELL; NORVIG, 1995);

- Habilidade social: os agentes interatuam com outros agentes;

- Reatividade: são capazes de perceber seu ambiente e responder às mudanças que ocorrem nele;

- Proatividade: os agentes não só atuam com seu ambiente, eles têm a capacidade de ter um comportamento dirigido a objetivos, tomando a iniciativa.

A fim de representar e conceber agentes que se diferenciam por suas características e habilidades, podem ser utilizadas ferramentas de programação com arquiteturas de software que facilitam a construção e concepção do comportamento de agentes em seus sistemas complexos. Existem vários tipos de arquiteturas 
de software de agentes, tais como (RUSSELL; NORVIG, 1995):

- Arquitetura Reativa: Os agentes não aprendem nem atualizam suas regras de comportamento, mas respondem passivamente a outros agentes e ao ambiente (ZECHMAN, 2015), com base em mecanismos estimulo-resposta.

- Arquitetura Cognitiva ou Deliberativa: Os agentes desenvolvem açóes ou tomam decisóes através de um raciocínio lógico. A arquitetura BDI (BRATMAN, 1987) (Believe, Desire, Intention) é muito utilizada onde o comportamento humano é modelado segundo as crenças, desejos e intençốes do agente.

- Arquitetura Híbrida: combina componentes das arquiteturas reativas e deliberativas.

Russell e Norvig (2002) também sugerem a caracterização de agentes por meio da metodologia denominada de PAGE (acrônimo para Perceptions, Actions, Goals e Environment). Nesse caso o agente percebe estímulos do ambiente por meio de sensores; as açôes (decisões) são consequências das percepçóes que são utilizadas para alcançar um objetivo.

O ambiente é o mundo, representado de forma virtual, onde atuam os agentes. $\mathrm{O}$ ambiente pode ser representado por espaços geográficos (como o caso de cidades, bairros, moradia) ou por locais onde os agentes atuam e tomam decisóes (GILBERT, 2007).

Russell e Norvig (1995) classificam os ambientes da seguinte forma:

- Acessível vs. Inacessível: No ambiente acessível o agente consegue obter informação atualizada e completa do ambiente.

- Determinístico Vs Não determinístico: no ambiente determinístico a ação selecionada pelo agente determina o próximo estado do ambiente, cada ação tem um efeito único sem incertezas nos resultados.

- Estático e Dinâmico: o ambiente estático não se altera, enquanto o ambiente dinâmico molda-se de acordo com a interação com os agentes.
- Discreto Vs Contínuo: É discreto quando o número de percepçóes e açóes são finitos.

Para realizar a modelagem baseada em agentes faz-se necessária a construção de um modelo conceitual que permita ter uma visão do comportamento dos agentes, da interação entre eles e seu ambiente como também das motivaçóes para a tomada de decisóes. Existem algumas metodologias disponíveis para construção de plataformas (ou sistemas de software) de modelagem com uso de agentes, tais como a Metodologia Gaia (WOOLDRIDGE; JENNINGS; KINNY, 2000); a MESSAGE (ZAMBONELLI; JENNINGS; WOOLDRIDGE, 2003), UML (BAUER; MÜLLER; ODELL, 2001) dentre outros. A Metodologia TROPOS (BRESCIANI et al., 2004) cujos princípios de modelagem são utilizados neste artigo, é uma metodologia que realiza a modelagem dos atores, de dependências, de metas, de planos e de competências em MBA.

As noçóes mentalísticas da metodologia TROPOS são baseadas na arquitetura BDI (Believe, Desire, Intention) que se baseia nos seguintes conceitos e relacionamentos (BRESCIANI et al., 2004):

- Ator (agente): é uma entidade que tem objetivos estratégicos e intençóes dentro do sistema representados por meio deum papel e uma posição.

- Objetivo (meta): representa os interesses estratégicos dos atores que também podem ser expressos como desejos. Os objetivos são divididos em: objetivo que é o estado futuro que o ator deseja alcançar e objetivos-suaves (softgoals) que são difíceis de afirmar se serão alcançados.

- Plano: Representa a maneira como serão realizadas as açóes (a estratégia de ação) para satisfazer algum objetivo.

- Recurso: são entidades físicas ou de informação que podem ser disponibilizados aos atores para realizar uma ação.

- Dependência: entre dois atores indica que um ator depende de outro, por algum motivo, para alcançar algum objetivo, executar algum plano ou entregar ou usar um recurso. 
- Capacidade: representa a habilidade de um ator de definir, escolher e executar um plano para o cumprimento de um objetivo.

- Crença: representa o conhecimento e a percepção dos agentes em relação ao mundo ou ao ambiente.

\section{APLICAÇÓES DE MODELOS BASEADOS EM AGENTES PARA GESTÁO DE SISTEMAS HÍDRICOS}

Atualmente, tem aumentado a publicação de trabalhos com aplicação de modelos baseados em agentes para inserção do componente social às simulaçóes do comportamento de sistemas ambientais para representar a influência da interação socioambiental na implementação de diferentes alternativas de gestão. Nesse contexto de modelagem sócio ambiental e para o caso de sistemas hídricos, os MBA podem contribuir par obter informaçôes sobre o nível de adaptação dos usuários frente a diferentes políticas ou açóes de gestão que busquem melhorar indicadores de performance do sistema bem como o nível de satisfação dos usuários.

Os trabalhos desenvolvidos na área de gestão dos recursos hídricos utilizando MBA também evidenciam a importância da articulação da gestão do recurso hídrico com a gestáo do solo e recursos conexos para solucionar os conflitos relativos ao sistema de uma maneira adaptativa, considerando o agente social atuante no sistema hídrico e, portanto, capaz de alterar o estado do sistema por meio de seus hábitos e comportamentos. O agente social ao tempo em que atua no sistema hídrico (com seus requisitos de demanda e padrôes de consumo) sofre também os efeitos de falhas que o sistema possa apresentar.

Existem alguns trabalhos que utilizam os modelos baseados em agentes na área da gestão de recursos hídricos, para explorar mecanismos de avaliação de performance dos sistemas hídricos (SCHLÜTER; PAHL-WOSTL, 2007), entender melhor a complexidade dos usos da água e dos usuários da água em sub-bacias (BERGER et al., 2006), representar a dinâmica do uso da água para irrigação e o efeito que tem sobre a distribuição espacial e temporal dos recursos hídricos (VAN OEL et al., 2010), avaliação de estratégias alternativas de operação de reservatórios na distribuição do uso da água (VAN OEL; KROL; HOEKSTRA, 2012); avaliação das consequências do comportamento dos agricultores na qualidade da água (NG et al., 2011; MAZZEGA et al., 2014), integração de ferramentas para análises da gestão integrada da água (NIKOLIC; SIMONOVIC; MILICEVIC, 2013); uso e manejo da água (AKHBARI; GRIGG, 2013, 2014, 2015; HOLTZ; PAHL-WOSTL, 2012; BELAQZIZ et al., 2013; FARHADI et al., 2016) governança e política de uso da água (WISE; CROOKS, 2012; GIULIANI; CASTELLETTI, 2013; MULLIGAN, et al., 2014; CASTILLA-RHO et al., 2015; LECLERT; NZIOKI; FEUERSTEIN, 2016; HAN et al., 2017), dentre outros.

\section{Modelos baseados em agentes para gerenciamento de sistemas urbanos de abastecimento de água}

O presente trabalho insere-se no contexto da aplicação de MBA para a gestão de sistemas hídricos urbanos, especialmente em situaçóes de restrição hídrica. A efetividade do gerenciamento de sistemas urbanos de abastecimento de água em situação de estresse hídrico apresenta-se especialmente atrelada à gestão da demanda urbana de água.

Medidas e açốes impostas em Planos Emergenciais podem alcançar diferentes níveis de efetividade conforme a adesão e a resposta apresentada por usuários do sistema. Campanhas educativas, rodízio ou racionamento do abastecimento, tarifas de contingência ou bonificaçóes por redução de consumo são exemplos de medidas cujas efetividades estáo diretamente relacionadas ao comportamento do usuário do sistema.

Tais medidas buscam atenuar as consequências de um período prolongado de escassez hídrica, porém é importante considerar que a adequada adoção (aceitaçáo) dessas estratégias por parte da população pode afetar o resultado esperado ou sua efetividade. Um modelo baseado em agentes que represente essa situação pode contar com agentes em diferentes papéis, tais como usuários comerciais, usuários residenciais, companhias de abastecimento, agências reguladoras, comitês de bacias hidrográficas, associaçôes civis, dentre outros. A gestão da água para consumo humano é resultado emergente dos atores que se relacionam afetando as decisóes individuais e coletivas. 
Considerar os sistemas hídricos como sistemas complexos adaptativos-SCA permite visualizar a interação entre o crescimento populacional, a mudança de uso do solo, os processos hidrológicos, o consumo residencial de água e as práticas conservacionistas (GIACOMONI; ZECHMAN, 2010) ou simular estratégias de consumo e conservação de água, segundo as demandas do recurso (KANTA; ZECHMAN, 2014) como também para avaliar estratégias adaptativas na gestão dos recursos hídricos.

Existem vários estudos que utilizam MBA para o desenvolvimento de políticas relacionadas a sistemas de abastecimento urbano onde são avaliadas as dinâmicas de entre políticas de recursos hídricos e políticas de recursos conexos (DUCROT et al., 2004; GIACOMONI; KANTA; ZECHMAN, 2013; KANTA; ZECHMAN, 2014), avaliação de políticas de água alternativas em diferentes cenários (LÓPEZ-PAREDES; SAURÍ; GALÁN, 2005; YAN; YUNFENG, 2010), avaliação e otimização de demanda/oferta de água residencial (ATHANASIADIS et al., 2005; TSEGAYE; VAIRAVAMOORTHY, 2009; LINKOLA; ANDREWS; SCHUETZE, 2013; YUAN et al., 2014; ALI; SHAFIEE; BERGLUND, 2017; KOUTIVA; MAKROPOULOS, 2016) gerenciamento e governança da água (ALI et al., 2014; CHU et al., 2009), avaliação de comportamento de usuários da água (EDMONDS; BARTHELENY; MOSS, 2002; GUNKEL; KÜLLS, 2006; RIXON; BURN, 2007), alocação do recurso hídrico (DING et al., 2016), dentre outros.

\section{Proposta de MBA para gerenciamento de sistema urbano de abastecimento de água}

A seguir é apresentada uma proposta de concepção de MBA para gerenciamento de sistema urbano de abastecimento de água que incorpore o comportamento do usuário de água na avaliação de alternativas de gestão de demanda. O modelo busca ilustrar a influência do comportamento de consumidores e de sua capacidade de adaptação na efetividade de medidas de gestão da demanda em situaçôes de estresse hídrico em sistemas urbanos de abastecimento de água.

Para conceber e parametrizar o modelo, foi tomada como caso de estudo a região de Brazlândia, no Distrito Federal. O sistema de abastecimento de água (SAA) de Brazlândia tem como manancial os córregos Capão da Onça e Barracão. A quadra chuvosa na regiáo distribui-se entre o mês de outubro a abril, enquanto a estação seca prolonga-se de maio a setembro apresentando umidades bastante reduzidas chegando próximo a $12 \%$ em dias mais secos. No período de janeiro de 2017 a junho de 2018, o Distrito Federal passou por uma séria crise de escassez hídrica com seu principal reservatório de abastecimento o Descoberto atingiu níveis de reservação em torno de $9 \%$ de sua capacidade nos momentos mais críticos. Tal situação motivou medidas e açôes de gestão objetivando reduzir a demanda e também medidas que buscaram expandir a oferta por meio da construção de novas captaçóes no córrego Bananal e no Lago Paranoá. Dentro do contexto das medidas de gestáo de demanda, foi definido um sistema de rodízio no abastecimento (racionamento), foram veiculadas campanhas educativas para incentivar a redução do consumo e por, um período limitado, foi cobrado uma tarifa de contingência que penalizava consumidores que não alcançassem um determinado nível de redução de consumo estipulado pela ADASA (Agência Reguladora de Águas, Energia e Saneamento Básico do Distrito Federal). A fim de avaliar a influência do comportamento do usuário na efetividade das medidas de gestão, foi selecionada a campanha educativa como medida de gestão a ser modelada. Como complemento à reaçáo do consumidor frente às campanhas educativas, foi avaliada sua percepçáo em relação ao período de chuva. Ou seja, buscava-se avaliar como a percepção do consumidor diante de eventos de chuva influenciava o nível de consumo.

$\mathrm{Na}$ concepção do modelo, cada agente representa um domicílio (casa) que tem um certo nível de consumo residêncial de água. Os agentes residenciais foram categorizados em agentes de renda baixa, média e alta e podiam comportar-se de forma cooperativa ou não cooperativa. Os agentes foram classificados em:

- Agente Não Cooperativo: Não se adapta às regras de consumo de água estabelecidas pelo gestor, não diminui a quantidade de água consumida, podendo ainda, aumentar o consumo.

- Agente Cooperativo: adapta-se às regras de consumo de água estabelecidas pelo gestor, diminui a quantidade de água consumida. 
Tomou-se como hipótese que o comportamento dos agentes residenciais poderia ser influenciado pela percepção do agente em relação à disponibilidade de água durante períodos chuvosos e secos e à temperatura do ambiente.

Os agentes são descritos como reativos e suas características e estratégias de ação foram construídas mediante a metodologia PAGE (Perceptions, Actions, Goals, Environmental). Para construir o PAGE que daria suporte à parametrização das decisóes e comportamentos dos agentes no modelo, foram realizadas entrevistas com consumidores domiciliares de água da área urbana de Brazlândia. As entrevistas foram realizadas in loco por meio de visitas aos domicílios e com base em questionário que tinha como foco a identificação de informaçóes sobre o padrão de uso da água, a percepção de consumo e quantidades de água consumidas nas atividades cotidianas, a percepção em relação às campanhas educativas para conservação do uso da água e o nível de redução de consumo motivado por essas campanhas (veiculadas pela Companhia de Saneamento e Esgoto do Distrito Federal-CAESB e a ADASA). O questionário também continham perguntas que investigavam a percepção dos consumidores em dias de chuva e períodos de seca, tentando estabelecer se o padrão de consumo era alterado em função de falsa noção de disponibilidade de água resultante de dias chuvosos. No total foram realizadas 173 entrevistas em 5 bairros distribuídos espacialmente e diferenciados pelo nível de renda, conforme ilustrado na Figura 1.

Na Tabela 1 são descritas as percepçóes (Perceptions) e ações (Actions) dos agentes em relação a campanhas educativas que tinha como objetivo promover a redução do consumo de água em áreas urbans A Tabela 1 resume informações obtidas nos questionários e entrevistas

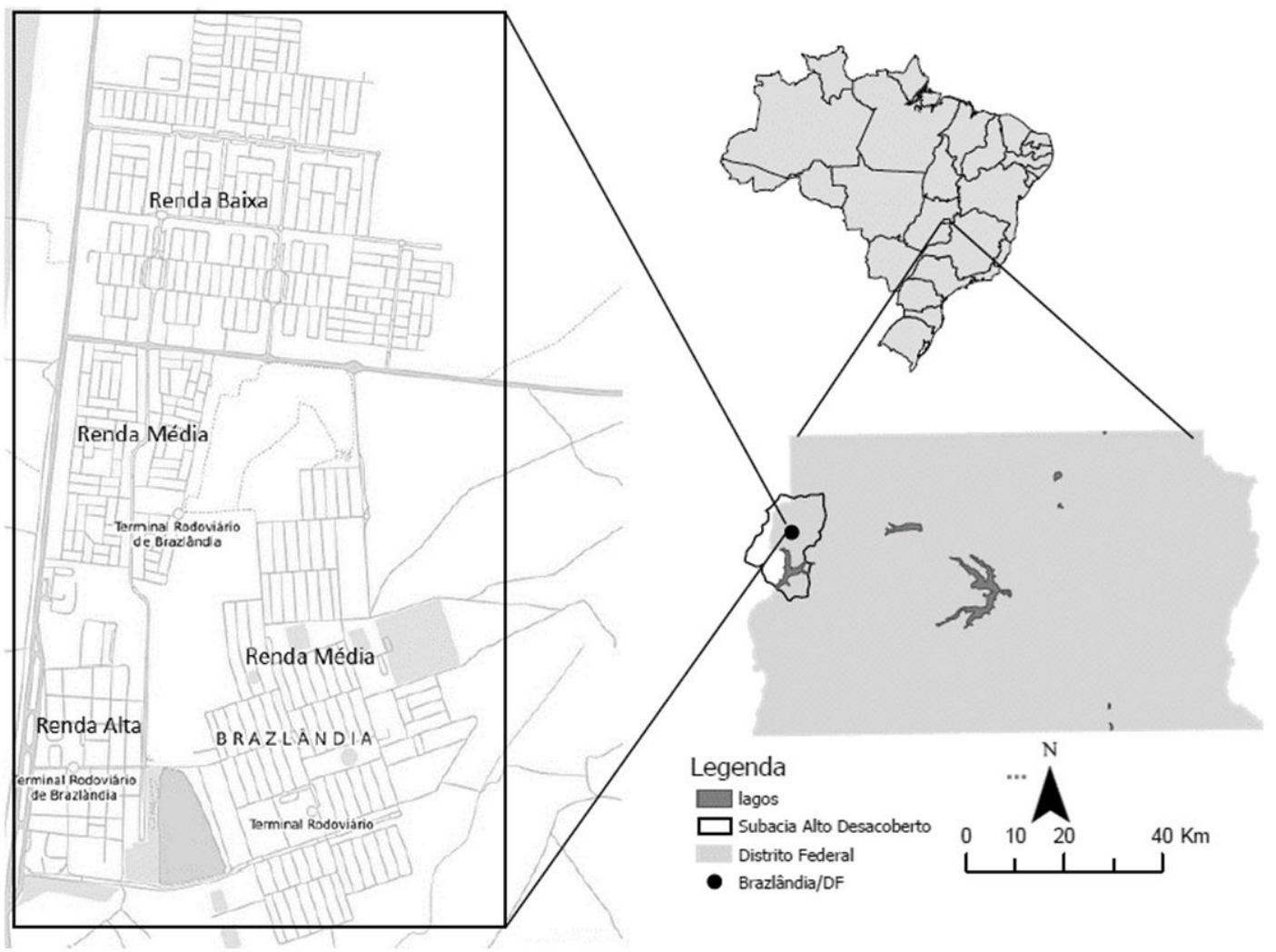

Figura 1- Localização de Brazlândia-DF e distribuição espacial do nível renda. 
e apresenta valores médios de consumo de água das faturas dos usuários e a porcentagem dos usuários que apresentaram consumos abaixo do valor médio.

$\mathrm{Na}$ Tabela 2 são apresentadas as percepçóes dos agentes (por categoria de renda) em relação ao clima e sua influência no consumo médio. Os valores referem-se à porcentagem dos agentes que apresentaram consumo de água mensal acima da média de consumo para cada categoria de renda. Percebe-se que em todas as categorias de renda, há um acréscimo no consumo de água durante o período de seca. No período de seca, mais de $70 \%$ da população apresentou consumo de água acima da média em todas as categorias de renda, o que reforça a relação entre a percepção do clima e o nível de consumo. No entanto, esse padrão de comportamento náo apresentou especificidade para nenhuma das categorias de renda, ou seja, é um comportamento padrão em todas as rendas.

A representação do ambiente (Environmental) é a mesma para todos os agentes. As características do ambiente foram descritas como:

- Parcialmente observável;

- Estático: para cada step de simulação;

Tabela 1- Percepções e Açôes dos agentes em relação às campanhas educativas.

\begin{tabular}{ccc}
\hline Renda & $\begin{array}{c}\text { Consumo médio } \\
\left(\mathbf{m}^{3} / \mathbf{m e ̂ s}\right)\end{array}$ & $\begin{array}{c}\text { \% pop consumo } \\
\text { abaixo da média }\end{array}$ \\
\hline Baixa & 16,9 & 80,46 \\
Media & 17,8 & 77,22 \\
Alta & 17,4 & 71,43 \\
\hline
\end{tabular}

Tabela 2- Percepçôes e açóes dos agentes em relação ao clima.

\begin{tabular}{ccc}
\hline Renda & $\begin{array}{c}\text { Percepçáo do } \\
\text { Clima }\end{array}$ & $\begin{array}{c}\text { \% pop consumo } \\
\text { acima da média }\end{array}$ \\
\hline Baixa & Chuva & 5,75 \\
& Seca & 77,01 \\
Média & Chuva & 7,59 \\
& Seca & 74,68 \\
Alta & Chuva & 14,29 \\
& Seca & 71,43 \\
\hline
\end{tabular}

- Dinâmico pelo consumo de água;

- Não determinístico;

- Discreto;

- Episódico (mês);

- Tamanho: multiagente.

O intervalo de tempo considerado na simulação do modelo (step) é mensal e considera a época de chuva e época sem chuva, dado que em Brazlândia o período de chuva é bem definido nos meses de outubro a abril. Todos os agentes têm um mesmo objetivo (Goal) que é "Satisfazer as necessidades mensais de uso da água".

Os elementos que compóem os diagramas resultantes da aplicação da metodologia TROPOS para ilustração do comportamento dos agentes estâo exemplificados na Figura 2. Nas Figuras 3 e 4 são mostrados os comportamentos do agente residencial cooperativo (A.R.C) e o agente residencial não cooperativo (A.Nc) para cada tipo de renda.

Os consumos médios mensais dos agentes são: $16,9 \mathrm{~m} 3$ para renda baixa, $17,8 \mathrm{~m} 3$ para renda média e 17,4 m3 para renda alta (Tabela 1). Na Figura 3 é apresentado o modelo conceitual para renda baixa e média pois, de acordo com os resultados das entrevistas e dos questionários, o comportamento de consumo de água é similar para essas rendas. Por meio das visitas aos domicílios, verificou-se que o armazenamento de água em épocas de chuva é um hábito costumeiro em Brazlândia, principalmente nas rendas média e baixa, denotando uma boa receptividade ao aproveitamento de água de chuva. A Figura 3 ilustra esse comportamento,

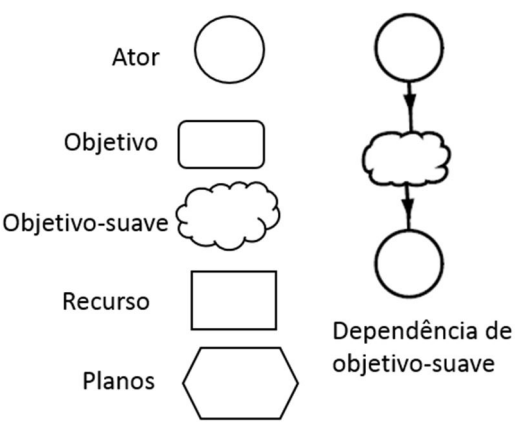

Figura 2- Diagramas da metodologia Tropos, modificado de Bresciani et al. (2004) 


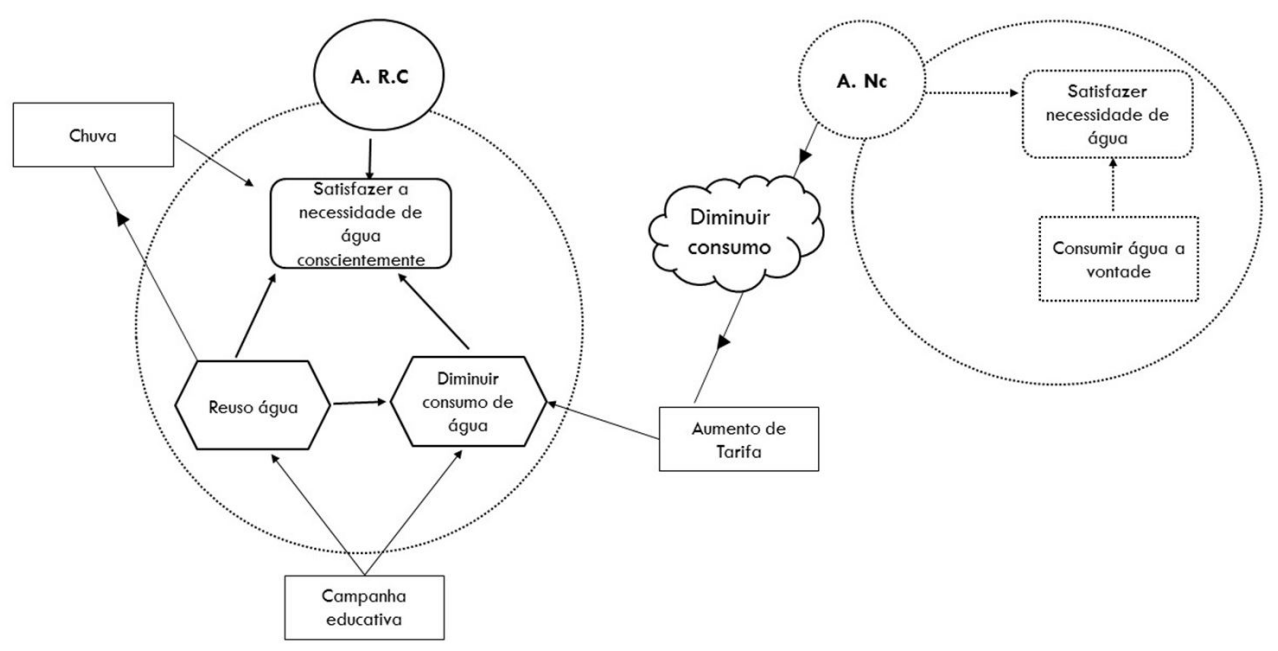

Figura 3- Modelo Conceitual do Comportamento de Agentes de Renda Baixa e Média

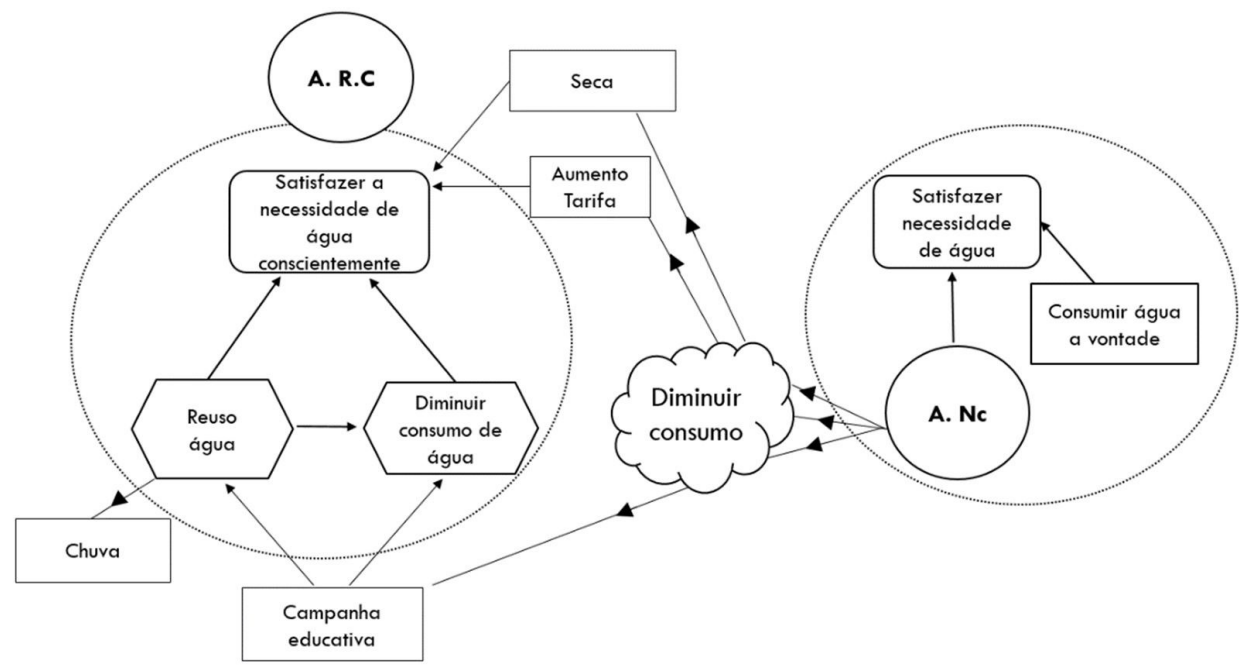

Figura 4- Modelo Conceitual do comportamento de Agentes de Renda Alta

evidenciando que o consumo é menor para os três tipos de rendas, porém a renda baixa e média têm uma porcentagem maior de casas que consumem água embaixo do consumo médio em épocas de chuva, mostrando maior economia nessa época por parte do $6 \%$ a mais dos agentes das rendas baixa e média, comparado com a renda alta.

No caso dos agentes não cooperativos (renda baixa e média), é apresentado um fator de dependência mediante um objetivo-suave denominado "diminuir consumo". Neste caso, o agente é influenciado pelo aumento de tarifa para satisfazer o objetivo-suave e se tornar cooperativo (Figura 3).

No caso da Figura 4 para renda alta, a época de seca e as campanhas educativas são considerados recursos para diminuir o desperdício de água. Para que o agente não cooperativo de renda alta se torne cooperativo serão necessárias campanhas educativas, 
época de seca e outras açóes de gestáo tais como o aumento de tarifas.

As Campanhas Educativas são consideradas como um recurso que motiva açóes cooperativas para diminuir o desperdício de água. Por meio das simulaçóes do MBA, foi verificado que os agentes de renda baixa e média apresentarm uma economia de 25 e $24 \%$ de água consumida respectivamente, por causa das campanhas educativas, já a renda alta economiza $18 \%$ da água. Esses resultados mostram a importância e influência que as campanhas educativas têm sobre o comportamento de consumo dos usuários da água.

$\mathrm{Na}$ época sem chuvas (seca) nos três tipos de renda, mais do $70 \%$ dos agentes consomem mais água que em época de chuva, este resultado era esperado pois no período seco as temperaturas aumentam e, portanto, as necessidades de água também, porém é um resultado preocupante pois verifica-se uma maior pressão sobre a disponibilidade hídrica num período sem chuva para recuperação das fontes hídricas e níveis dos mananciais.

Dentre os agentes que economizam água no período de seca, a renda média reduziram aproximadamente $11 \%$ do consumo de água, enquanto a renda alta e baixa, ambas economizaram em torno de 3\%. Por outro lado, na categoria de renda alta houve um aumento no número de agentes $(8,54 \%)$ que economizam água (consumo embaixo do consumo médio) nessa época, comparado com a categoria de renda baixa, que reduziu a quantidade de agentes com consumo abaixo da média na época da seca.

$\mathrm{O}$ aumento de tarifas foi também um recurso considerado para mudança do comportamento não cooperativo para cooperativo nos três tipos de renda pois, no estudo mostrou-se que 70,3\% dos agentes de renda baixa e $76,9 \%$ dos agentes de renda média diminuíram o consumo de água devido à implantação de uma tarifa de contingência. Para o caso da renda alta a tarifa também influenciou na diminuição do consumo, nesse caso para $55,6 \%$ dos agentes.

Nas três rendas também foi identificado que o reuso de água da máquina de lavar constitui-se uma atividade que favorece a diminuição do consumo de água, pois a maioria dos agentes em Brazlândia realiza esta atividade $(84,9 \%$ renda baixa, $82,1 \%$ renda média e 71,4\% renda alta) podendo-se considerar como um hábito dos agentes na região de Brazlândia.

\section{CONCLUSÓES}

Os sistemas que usam o recurso hídrico para diferentes destinos devem ser considerados como sistemas complexos devido às interaçóes dos seus componentes e às mudanças que estas interaçóes podem desenvolver no sistema.

Nesse contexto e devido às mudanças no sistema, a gestão dos recursos hídricos tem que ser considerada como adaptativa e não como um processo estático, pois os usuários podem alterar os processos e as políticas a serem implementadas pelos gestores.

Cresce na literatura o desenvolvimento de modelos para simular os conflitos de uso dos recursos hídricos considerando a representação do comportamento dos agentes sociais. Esses modelos possibilitam ter uma visão global do sistema, permitindo comparar políticas de gestão da água e seus impactos no comportamento e nas decisóes de usuários e outros atores.

No caso do uso dos Modelos Baseados em Agentes-MBA, para modelar sistemas hídricos, considerados como sistemas complexos adaptativos, as pesquisas têm como resultado um melhor entendimento da evolução sócio ambiental do sistema mediante a execução de estratégias de gestão adaptativa.

Para realizar este tipo de modelagem e gerar resultados satisfatórios na gestáo adaptativa dos recursos hídricos é indispensável a realização do modelo conceitual e a descrição detalhada do comportamento dos agentes e sua interação com o ambiente e entre eles, mediante o PAGE ou outras metodologias que permitam analisar as percepçôes e açóes dos agentes. Recomenda-se que para a construção do PAGE seja realizado trabalho em campo com os atores envolvidos para ter uma melhor percepção e representação dos comportamentos a serem analisados.

No presente trabalho foi realizada a simulação de sistemas de abastecimento de água em áreas urbanas como um sistema complexo adaptativo. $\mathrm{O}$ modelo construído facilitou o entendimento do comportamento do usuário frente a medidas de gestão de demanda por meio da execução de estratégias de gestão adaptativa representadas por campanhas educativas e pela implantação de tarifas.

Considerar o agente social como principal ator, analisar seu comportamento e como este pode afetar 
diretamente as políticas a serem implementadas e sua efetividade, permitirá estabelecer estratégias para melhorar a gestão dos recursos hídricos.

Para a comunidade de Brazlândia no Distrito Federal, foi importante considerar o tipo de renda dos consumidores de água como uma característica importante, pois esta tem influência direta no consumo de água podendo afetar o comportamento cooperativo e não cooperativo dos agentes. Os períodos de chuvas e seca também têm um peso importante nas decisōes dos agentes em Brazlândia, pois a presença de água no ambiente afeta a percepção do agente com relação à disponibilidade de água e como consequência afeta também seu consumo. No caso das campanhas educativas, pode-se considerar que tem uma participação importante nas atividades de diminuição de consumo, porém o aumento de tarifa tem maior influência nas mudanças de comportamento especialmente para rendas baixa e média.

Este tipo de resultados mostra a importância de considerar o comportamento do usuário e as condiçôes socioeconômicas na formulação das políticas de gestão de recursos hídricos, notadamente de no caso de sistemas hídricos urbanos que apresentam sensibilidade ao padrão de consumo da população. $\mathrm{O}$ trabalho apresentou a concepção de MBA para gestão de sistema hídrico urbano na tentativa de incorporar o comportamento e as decisóes dos consumidores como parte relevante do sistema e não apenas como input ou variável exógena. A evolução de MBA por meio de uma parametrização mais aderente à comunidade que tentam representar poderá auxiliar decisóes melhores informadas especialmente em períodos de escassez hídrica quando medidas de gestão direcionadas à redução do consumo possuem efetividade associada à cooperação ou não dos usuários do sistema.

\section{AGRADECIMENTOS}

Os autores agradecem ao Departamento de Ciências da Computação da Universidade de Brasília, especialmente à professora Célia Ghedini Ralha que ajudou no entendimento e com discussóes sobre a concepção dos sistemas multiagentes, e ao Cnpq pela bolsa concedida para um dos autores.

\section{REFERÊNCIAS}

AKHBARI, M.; GRIGG, N. S. A framework for an agent-based model to manage water resources conflicts. Water Resources Management, Cham, v. 27, n. 11, p. 4039-4052, 2013. http://dx.doi.org/10.1007/ s11269-013-0394-0.

AKHBARI, M.; GRIGG, N. S. Water management trade-offs between agriculture and the environment: a multiobjective approach and application. Journal of Irrigation and Drainage Engineering, New York, v. 140, n. 8, p. $1,2014$.

AKHBARI, M.; GRIGG, N. S. Managing water resources conflicts: modelling behavior in a decision tool. Water Resources Management, Cham, v. 29, n. 14 , p. 5201-5216, 2015. http://dx.doi.org/10.1007/ s11269-015-1113-9.

ALI, A. M. et al. A complex adaptive systems approach to simulate interactions among water resources, decision-makers, and consumers and assess urban water sustainability. In: INTERNATIONAL CONFERENCE ON HYDROINFORMATICS, 2014, New York. Proceedings... 2014.

ALI, A. M.; SHAFIEE, M. E.; BERGLUND, E. Z. Agent-based modeling to simulate the dynamics of urban water supply: climate, population growth, and water shortages. Sustainable Cities and Society, Amsterdam, v. 28, p. 420-434, 2017. http://dx.doi.org/10.1016/j.scs.2016.10.001.

ATHANASIADIS, N. et al. A hybrid agent-based model for estimating residential water demand. Simulation, San Diego, v. 81, n. 3, p. 175-187, 2005. http://dx.doi.org/10.1177/0037549705053172.

BATTY, M.; XIE, Y.; SUN, Z. Modeling urban dynamics through GISbased cellular automata. Computers, Environment and Urban Systems, New York, v. 23, n. 3, p. 205-233, 1999. http://dx.doi.org/10.1016/ S0198-9715(99)00015-0.

BAUER, B.; MÜLLER, J. P.; ODELL, J. Agent UML: a formalism for specifying multiagent software systems. International Journal of Software Engineering and Knowledge Engineering, Singapore, v. 11, n. 3, p. 207-230, 2001. http://dx.doi.org/10.1142/S0218194001000517.

BELAQZIZ, S. et al. An agent based modeling for the gravity irrigation management. Procedia Environmental Sciences, Amsterdam, v. 19, p. 804-813, 2013. http://dx.doi.org/10.1016/j.proenv.2013.06.089.

BERGER, T. et al. Capturing the complexity of water uses and water users within a multi-agent framework. Water Resources Management, Cham, v. 21, n. 1, p. 129-148, 2006. http://dx.doi.org/10.1007/s11269006-9045-z. 
BLASKOVICH, F. Synergy and emergence in energy policy design. In: SPE ASIAPACIFIC OIL \& GAS CONFERENCE AND EXHIBITION, 2014, Adelaide, Australia. Proceedings... 2014.

BONABEAU, E. Agent-based modeling: methods and techniques for simulating human systems. National Academy of Sciences of the United States of America, Washington, v. 99, n. 33, p. 7280-7287, 2002. Suplemento 3. http://dx.doi.org/10.1073/pnas.082080899.

BRATMAN, M. E. Intention, plans and practical reason. Cambridge: Havard University Press, 1987

BRESCIANI, P. et al. Tropos: an agent-oriented software development methodology. Autonomous Agents and Multi-Agent Systems, Norwell, v. 8, p. 203-236, 2004.

CASTILLA-RHO, C. et al. An agent-based platform for simulating complex human e aquifer interactions in managed groundwater systems. Environmental Modelling \& Software, Oxford, v. 73, p. 305-323, 2015. http://dx.doi.org/10.1016/j.envsoft.2015.08.018.

$\mathrm{CHU}$, J. et al. Agent-based residential water use behavior simulation and policy implications: a case study in Beijing city. Water Resources Management, Cham, v. 23, n. 15, p. 3267-3295, 2009. http://dx.doi. org/10.1007/s11269-009-9433-2.

DING, N. et al. Agent based modelling for water resource allocation in the transboundary Nile River. Water, Basel, v. 8, n. 4, p. 139, 2016.

DUCROT, R. etal. Articulating land and water dynamics with urbanization: an attempt to model natural resources management at the urban edge. Computers, Environment and Urban Systems, New York, v. 28, n. 1-2, p. 85-106, 2004. http://dx.doi.org/10.1016/S0198-9715(02)00066-2.

EDMONDS, B.; BARTHELENY, O.; MOSS, S. Domestic water demand and social influence: an agent-based modeling approach. 2002. CPM Report 02-103.

FARHADI, S. et al. An agent-based-nash modeling framework for sustainable groundwater management: a SCAe study. Agricultural Water Management, Amsterdam, v. 177, p. 348-358, 2016. http:/l dx.doi.org/10.1016/j.agwat.2016.08.018.

GIACOMONI, H.; KANTA, L.; ZECHMAN, E. Complex adaptive systems approach to simulate the sustainability of water resources and urbanizatio. Journal of Water Resources Planning and Management, New York, v. 139, n. 5, p. 554-564, 2013. http://dx.doi.org/10.1061/ (ASCE)WR.1943-5452.0000302.

GIACOMONI, M.; ZECHMAN, E. M. A complex adaptative systems approach to simulate urban water resources sustainability. In: WORLD ENVIRONMENTAL AND WATER RESOURCES CONGRESS 2010: CHALLENGES OF CHANGES, 2010. Proceedings... Providence, Rhode Island: ASCE, 2010.
GILBERT, N. Agent-based models. Guildford: University of Surrey, 2007.

GIULIANI, M. E.; CASTELLETTI, A. Assessing the value of cooperation and information exchange in large water resources systems by agentbased optimization. Water Resources Research, Washington, v. 49, n. 7, p. 3912-3926, 2013. http://dx.doi.org/10.1002/wrcr.20287.

GUNKEL, A.; KÜLLS, C. H. Towards agent-based modelling of stakeholder behaviour: a pilot study on drought vulnerability of decentral water supply in NE Brazil. In: IEMSS FOURTH BIENNIAL MEETING: INTERNATIONAL ENVIRONMENTAL MODELLING AND SOFTWARE SOCIETY, 2006, USA. Proceedings... 2006.

HAN, T. et al. Study on environment-economy-society relationship model of Liaohe River Basin based on multi-agent simulation. Ecological Modelling, Amsterdam, v. 359, p. 135-145, 2017. http:// dx.doi.org/10.1016/j.ecolmodel.2017.02.016.

HOLLAND, J. Hidden order: how adaptation builds complexity. USA: Addison Wesley, 1995. 185 p.

HOLTZ, G.; PAHL-WOSTL, C. An agent-based model of groundwater over-exploitation in the Upper Guadiana, Spain. Regional Environmental Change, New York, v. 12, n. 1, p. 95-121, 2012. http://dx.doi.org/10.1007/ s10113-011-0238-5.

KANTA, L.; ZECHMAN, E. Complex adaptive systems framework to assess supply-side and demand-side management for urban water resources. Journal of Water Resources Planning and Management, New York, v. 140, n. 1, p. 75-85, 2014. http://dx.doi.org/10.1061/(ASCE) WR.1943-5452.0000301.

KEUR, P. et al. Adaptative water management in terms of development and application within IWRM. In: The adaptative resource management handbook. London: Earthsca, 2010.

KOUTIVA, I.; MAKROPOULOS, C. Modelling domestic water demand: an agent based approach. Environmental Modelling \& Software, Oxford, v. 79, p. 35-54, 2016. http://dx.doi.org/10.1016/j.envsoft.2016.01.005.

LECLERT, L.; NZIOKI, R.; FEUERSTEIN, L. Addressing governance and management challenges in small water supply systems: the integrity management approach in Kenya. Aquatic Procedia, Amsterdam, v. 6, p. 39-50, 2016. http://dx.doi.org/10.1016/j.aqpro.2016.06.006.

LINKOLA, L.; ANDREWS, C.; SCHUETZE, T. An agent based model of household water use. Water, Basel, v. 5, p. 1082-1100, 2013.

LÓPEZ-PAREDES, A.; SAURÍ, D.; GALÁN, J. M. Urban water management with artificial societies of agents: the FIRMABAR simulator. Simulation, San Diego, v. 81, n. 3, p. 189-199, 2005. http://dx.doi. org/10.1177/0037549705053167. 
MACAL, C. M.; NORTH, M. J.; Tutorial on agent-based modeling and simulation. Journal of Simulation, Birmingham, v. 4, n. 3, p. 151-162, 2010. http://dx.doi.org/10.1057/jos.2010.3.

MAZZEGA, P. et al. Critical multi-level governance issues of integrated modelling: an example of low-water management in the Adour-Garonne basin (France). Journal of Hydrology, Amsterdam, v. 519, p. 2515-2526, 2014. http://dx.doi.org/10.1016/j.jhydrol.2014.09.043.

MITCHELL, M. Complex systems: network thinking. Portland State University, Santa Fe Institute, 2006.

MITLETON-KELLY, E. Complex systems and evolutionary perspectives of organisations: the application of complexity theory to organisations: advanced series in management. Oxford: Elsevier Science, 2003.

MULLIGAN, K. et al. Assessing groundwater policy with coupled economic-groundwater hydrologic modeling. Water Resources Research, Washington, v. 50, n. 3, p. 2257-2275, 2014. http://dx.doi. org/10.1002/2013WR013666.

NG, T. L. et al. An agent-based model of farmer decision-making and water quality impacts at the watershed scale under markets for carbon allowances and a second-generation biofuel crop. Water Resources Research, Washington, v. 47, n. 9, p. 1, 2011. http://dx.doi. org/10.1029/2011WR010399.

NIKOLIC, V.; SIMONOVIC, S.; MILICEVIC, D. B. Analytical support for integrated water resources management: a new method for addressing spatial and temporal variability. Water Resources Management, Cham, v. 27, n. 2, p. 401-417, 2013. http://dx.doi.org/10.1007/s11269-012-0193-z.

RAMMEL, C.; STAGL, S.; WILFING, H. Managing complex adaptative systems: a co-evolutionary perspective on natural resource management. Ecological Economics, Amsterdam, v. 63, n. 1, p. 9-21, 2007. http:// dx.doi.org/10.1016/j.ecolecon.2006.12.014.

RAND, W. Sistemas complexos: conceitos, literatura, possibilidades e limitações. In: FURTADO, B.; SAKOWSKI, P.; TÓVOLLI, P. (Orgs.). Modelagem de sistemas complexos para politicas públicas. Brasilia: IPEA, 2015. p. 43-64.

RIXON, A.; BURN, S. Exploring water conservation behaviour through participatory agent-based modelling. In: Topics on system analysis and integrated water resources management. Elsevier, 2007. cap. 4, p. 73-96.

RUSSELL, S.; NORVIG, P. Artificial intelligence: a modern approach. New Jersey: Prentice Hall, 1995. 931 p.

RUSSELL, S.; NORVIG, P. Artificial intelligence: a modern approach 2nd ed. USA: Prentice-Hall, 2002.
SCHLÜTER, M.; PAHL-WOSTL, C.; Mechanisms of resilience in common-pool resource management systems: an agent-based model of water use in a river basin. Ecology and Society, Ottawa, v. 12, n. 2, p. 4, 2007. http://dx.doi.org/10.5751/ES-02069-120204.

SICHMAN, J. Operacionalização de sistemas complexos. In: FURTADO, B.; SAKOWSKI, P.; TÓVOLLI, P. (Orgs.). Modelagem de sistemas complexos para políticas públicas. Brasília: IPEA, 2015.

TSEGAYE, S.; VAIRAVAMOORTHY, K. Agent-based modeling to estimate residential water demand and to explore optimal demand side water management strategies: water engineering development centre. Loughborough University, 2009.

VAN OEL, P. et al. Feedback mechanisms between water availability and water use in a semi-arid river basin: a spatially explicit multi-agent simulation approach. Environmental Modelling \& Software, Oxford, v. 25, n. 4, p. 433-443, 2010. http://dx.doi.org/10.1016/j.envsoft.2009.10.018.

VAN OEL, P.; KROL, M. S.; HOEKSTRA, A. Application of multi-agent simulation to evaluate the influence of reservoir operation strategies on the distribution of water availability in the semi-arid Jaguaribe basin, Brazil. Physics and Chemistry of the Earth, Oxford, v. 47-48, p. 173-181, 2012.

WISE, S.; CROOKS, A. T. Agent-based modeling for community resource management: acequia-based agriculture. Computers, Environment and Urban Systems, New York, v. 36, n. 6, p. 562-572, 2012. http://dx.doi. org/10.1016/j.compenvurbsys.2012.08.004.

WOOLDRIDGE, M.; JENNINGS, N. R. Intelligent agents: theory and practice. The Knowledge Engineering Review, Cambridge, v. 10, n. 2, p. 115-152, 1995. http://dx.doi.org/10.1017/s0269888900008122.

WOOLDRIDGE, M.; JENNINGS, N. R.; KINNY, D. The Gaia methodology for agent-oriented analysis and design. Autonomous Agents and MultiAgent Systems, Norwell, v. 3, n. 3, p. 285-312, 2000. http://dx.doi. org/10.1023/A:1010071910869.

YAN, M. A.; YUNFENG, L. I. Conceptual research on decision making meetings for urban water management. IRSPSD International, Kanazawa, v. 3 , n. 3, p. $16-24,2010$.

YUAN, X.-C. et al. Urban household water demand in beijing by 2020: an agent-based model. Water Resources Management, Cham, v. 28, n. 10, p. 2967-2980, 2014. http://dx.doi.org/10.1007/s11269-014-0649-4.

ZAMBONELLI, F.; JENNINGS, N. R.; WOOLDRIDGE, M. Developing multiagent systems: the gaia methodology. ACM Transactions on Software Engineering and Methodology, New York, v. 12, n. 3, p. 317-370, 2003.

ZECHMAN, E. Using agent-based modeling for water resources planning and management. Journal of Water Resources Planning and Management, New York, v. 141, n. 11, 2015. 
Alves, C. M. A.; Herrera, D. J. M. $\cong$ Contribuiçöes de Modelos Baseados em Agentes (MBA)...

Diana Jimena Monsalve Herrera

Universidade de Brasília. dianajmonsalve@gmail.com

Contribuição do autor:

Definição do modelo conceitual, aplicação da metodologia incluindo aplicação dos questionários, análise dos resutlado e redação do artigo.

Conceiçáo de Maria Albuquerque Alves

Universidade de Brasília. cmaalves@gmail.com

Contribuição do autor:

Definição do objetivo e metodologia da pesquisa, revisão do texto e análise dos resultados. 